\title{
Uso da infusão de folhas de Sonchus oleraceus para o tratamento de vitiligo
}

\author{
Use of the infusion of Sonchus oleraceus leaves for the treatment of vitiligo \\ Uso de la infusión de hojas de Sonchus oleraceus para el tratamiento del vitiligo
}

Recebido: 12/03/2021 | Revisado: 18/03/2021 | Aceito: 21/03/2021 | Publicado: 28/03/2021

\author{
Gessi Somine Baron Eibel \\ ORCID: https://orcid/org/ 0000-0002-1757-898X \\ Universidade Estadual do Oeste do Paraná, Brasil \\ E-mail: simonieibel@hotmail.com \\ Adriana Zilly \\ ORCID: https://orcid/org/ 0000-0002-8714-8205 \\ Universidade Estadual do Oeste do Paraná, Brasil \\ E-mail: aazilly@hotmail.com \\ Rosane Meire Munhak da Silva \\ ORCID: https://orcid/org/ 0000-0003-3355-0132 \\ Universidade Estadual do Oeste do Paraná, Brasil \\ E-mail: zanem2010@hotmail.com \\ Helder Ferreira \\ ORCID: https://orcid.org/0000-0002-1089-6280 \\ Universidade Estadual do Oeste do Paraná, Brasil \\ E-mail: dr.helderferreira@gmail.com
}

\begin{abstract}
Resumo
Objetivo: Descrever o resultado do uso da infusão de folhas de Sonchus oleraceus para o tratamento de vitiligo. Método: Estudo qualitativo, realizado em um município de fronteira do Estado do Paraná, no segundo semestre de 2019 e início de 2020, com indivíduos que faziam uso da infusão da Sonchus oleraceus. Utilizou-se um instrumento semiestruturado com questões referentes à utilização da referida planta. Realizaram-se entrevistas, com análise temática dos resultados. Resultados: Foram participantes 11 indivíduos portadores de vitiligo, dos quais sete mulheres e quatro homens, com idade entre 25 a 61 anos (média de 42 anos), apresentando despigmentação em várias regiões do corpo. Faziam uso da infusão das folhas da Sonchus oleraceus no período de 3 a 13 meses (média de 9 meses), e preparavam o chá com 5 folhas em um litro de água, consumindo em média $700 \mathrm{~mL}$ ao dia. A maioria dos indivíduos observou pigmentação das áreas despigmentadas. A pigmentação das áreas foi observada no período entre 3 e 6 meses após o início do uso da infusão (média de 4 meses). Relacionada a doença os participantes expressaram tristeza, aborrecimento e diferença, com impacto direto na vida cotidiana, mas com a melhora após o uso da planta, felicidade e satisfação abriram espaço para uma nova vida. Conclusão: A infusão da Sonchus oleraceus ocasionou a pigmentação das manchas brancas de portadores de vitiligo, e os resultados da terapia fitoterápica proferiu sentimentos positivos aos participantes.
\end{abstract}

Palavras-chave: Vitiligo; Sonchus; Serralha; Plantas medicinais.

\begin{abstract}
Objective: To describe the results of using the infusion of Sonchus oleraceus leaves for the treatment of vitiligo. Method: Qualitative study, carried out in a border municipality in the State of Paraná, in the second half of 2019 and early 2020, with individuals who used the infusion of Sonchus oleraceus. A semi-structured instrument was used with questions regarding the use of that plant. Interviews were conducted, with thematic analysis of the results. Results: Participants were 11 individuals with vitiligo, seven women and four men, aged between 25 and 61 years (mean of 42 years), with depigmentation in various regions of the body. They used the infusion of the leaves of Sonchus oleraceus in the period from 3 to 13 months (average of 9 months), and prepared the tea with 5 leaves in a liter of water, consuming an average of $700 \mathrm{~mL}$ a day. Most individuals observed pigmentation in depigmented areas. The pigmentation of the areas was observed between 3 and 6 months after the start of the infusion (mean of 4 months). Related to the disease, the participants expressed sadness, boredom and difference, with a direct impact on daily life, but with the improvement after the use of the plant, happiness and satisfaction opened up space for a new life. Conclusion: The infusion of Sonchus oleraceus caused the pigmentation of white spots in patients with vitiligo, and the results of herbal therapy gave positive feelings to the participants.
\end{abstract}

Keywords: Vitiligo; Sonchus; Milkweed; Medicinal plants.

\section{Resumen}

Objetivo: Describir los resultados del uso de la infusión de hojas de Sonchus oleraceus para el tratamiento del vitiligo. Método: Estudio cualitativo, realizado en un municipio fronterizo del Estado de Paraná, en el segundo semestre de 
2019 y principios de 2020, con individuos que utilizaron la infusión de Sonchus oleraceus. Se utilizó un instrumento semiestructurado con preguntas sobre el uso de esa planta. Se realizaron entrevistas con análisis temático de los resultados. Resultados: Los participantes fueron 11 individuos con vitiligo, siete mujeres y cuatro hombres, con edades entre 25 y 61 años (media de 42 años), con despigmentación en varias regiones del cuerpo. Utilizaron la infusión de las hojas de Sonchus oleraceus en el período de 3 a 13 meses (promedio de 9 meses), y prepararon el té con 5 hojas en un litro de agua, consumiendo un promedio de $700 \mathrm{~mL}$ al día. La mayoría de las personas observaron pigmentación en áreas despigmentadas. La pigmentación de las áreas se observó entre 3 y 6 meses después del inicio de la infusión (media de 4 meses). Relacionado con la enfermedad, los participantes expresaron tristeza, aburrimiento y diferencia, con un impacto directo en la vida diaria, pero con la mejora luego del uso de la planta, la alegría y la satisfacción abrieron espacio para una nueva vida. Conclusión: La infusión de Sonchus oleraceus provocó la pigmentación de las manchas blancas en pacientes con vitiligo, y los resultados de la terapia a base de hierbas dieron sentimientos positivos a los participantes.

Palabras clave: Vitiligo; Sonchus; Algodoncillo; Plantas medicinales.

\section{Introdução}

O uso de plantas medicinais no tratamento de doenças é uma estratégia antiga, comprovada historicamente por registros de longa data, utilizada por praticamente todas as populações do mundo com o objetivo de promover e manter a saúde humana (Oliveira et al., 2017).

O Ministério da Saúde (MS) com o objetivo principal de garantir a prevenção de agravos, criou a Política Nacional de Plantas Medicinal e Fitoterápico (PNPMF) e a Política Nacional de Práticas Integrativas e Complementares (PNPIC) no Sistema Único de Saúde (SUS) (Ministério da Saúde, 2006a; Ministério da Saúde, 2006b).

Estas políticas incentivam o uso de plantas medicinais e fitoterápicos na Atenção Primária de Saúde (APS) promovendo e reconhecendo as práticas populares e tradicionais de uso de plantas medicinais, fitoterápicos e remédios caseiros que abrangem uma ampla utilização de plantas para fins terapêuticos, preparados nas casas dos consumidores (Mattos et al., 2018).

A inserção do uso de plantas medicinais nas Unidades de APS tem sido defendido e incentivado, mesmo porque, segundo a Organização Mundial de Saúde (OMS), 80\% da população mundial faz uso de plantas medicinais e seus preparos, as adquirindo em feiras livres, mercados populares, cultivadas nas casas ou na forma de extratos brutos para prevenção e tratamento de doenças (Zeni et al., 2017). Esta inserção pode transcender a incorporação de mais uma alternativa terapêutica à disposição do profissional de saúde, representando a junção entre o conhecimento popular e científico (Mattos et al., 2018).

No exterior e no Brasil, pesquisas evidenciaram que as plantas medicinais podem ser fontes de substâncias capazes de auxiliar no tratamento de inúmeras doenças como, por exemplo, o vitiligo (Doulah et al., 2020; Rodriguez et al., 2020).

Vitiligo é uma doença sistêmica crônica, adquirida, caracterizada pela despigmentação gradativa na pele, que afeta até $3 \%$ da população mundial, causada pela diminuição de células produtoras de melanina (melanócitos) provocando assim o aparecimento de manchas brancas por toda a pele e mucosas, de diferentes formas e tamanho, aumentando cada vez mais sua extensão. A região afetada, ou seja, área despigmentada fica mais sensível à luz solar deixando o portador mais vulnerável a outras doenças de pele (Kappor et al., 2020). Além da despigmentação, essa doença influencia profundamente a saúde emocional do portador, causando distúrbios psicológicos em grande parte deles, ocasionando estresse, ansiedade, depressão e baixo autoestima por não se sentirem autoconfiantes devido a sua aparência (Simons et al., 2020).

Existem diversos tratamentos para o vitiligo com o objetivo de estimular a produção de melanina no local afetado, como a micropigmentação, helioterapia, radiação ultravioleta, fenilalanina tópica e sistêmica e plantas medicinais. Além disso, há procedimentos invasivos que os pacientes são submetidos como a cirurgia de enxerto ou transplante de pele do próprio portador de uma região não afetada para a região afetada pela mancha, com o objetivo que esse tecido saudável que apresenta uma maior quantidade de melanócitos se desenvolva no local, fazendo com que a mancha desapareça (Dalla et al., 2020; Shah et al., 2019). 
Com respeito as plantas medicinais, a Sonchus oleraceus (SO) é utilizada empiricamente pela população para o tratamento do vitiligo, mas pouco se sabe sobre seu efeito sobre essa patologia. A SO é da família das Asteraceae, nativa do Brasil, popularmente conhecida como Serralha, possui tradicionalmente ação no tratamento de doenças hepáticas, do trato gastrointestinal e anemia (Nonato et al., 2018).

Diante do exposto e do desejo de fomentar o desenvolvimento de pesquisas em saúde pública com o intuito de promover a qualidade de vida da população, este estudo objetivou descrever o resultado do uso da infusão de folhas de Sonchus oleraceus para o tratamento de vitiligo.

\section{Metodologia}

Trata-se de um estudo de abordagem qualitativa, que buscará dar significados ao fenômeno estudado por meio da interpretação, cujo objeto não será mensurar os resultados, mas sim descrevê-los na integralidade (Pereira et., 2018).

O estudo foi desenvolvido em um município brasileiro de fronteira, localizado na região Oeste do Estado do Paraná. Neste município se encontra um horto de plantas medicinais, que tem o principal objetivo de implantação de um modelo de utilização de plantas medicinais e fitoterápicos no SUS, assim, cultiva inúmeras plantas medicinais, doa a população e produz fitoterápicos para as Unidades Básicas de Saúde aos municípios pertencentes a 9a regional de saúde do Paraná. Em dias préestabelecidos, inúmeros indivíduos procuram o horto para retirar plantas medicinais para prevenção e cura de enfermidades.

Foram incluídos no estudo os indivíduos com mais de 18 anos, com diagnóstico médico de vitiligo, que aceitaram participar da pesquisa e faziam uso da infusão da planta SO previamente ao início da pesquisa. Excluíram-se três indivíduos, deste modo, fizeram parte do estudo 11 indivíduos que atenderam os critérios de inclusão.

Os indivíduos que procuraram o horto, no segundo semestre de 2019, para retirar a planta SO para o tratamento de vitiligo, foram convidados a participar da pesquisa, na entrada do horto, em espaço público. A coleta de dados foi realizada por uma acadêmica de enfermagem de uma instituição pública de ensino, a qual recebeu treinamento prévio para a realização das entrevistas.

Utilizou-se um instrumento semiestruturado contendo questões referentes à utilização da SO, sobre sua finalidade de uso, como conheceu a planta, tempo de uso, quantidade utilizada e sentimentos por ser portador de vitiligo. As entrevistas foram gravadas em áudio e transcritas na íntegra posteriormente para análise. Na transcrição, as falas dos participantes foram identificadas pela letra P (participante) e pelo número consecutivo das entrevistas, por exemplo P1 (participante 1).

Os dados referentes as características apresentadas e a forma de tratamento foram organizados em um quadro demonstrativo, enquanto que, para a descrição dos sentimentos utilizou-se a análise temática dos dados, organizada em três fases: pré-análise, exploração dos dados coletados e tratamento dos resultados e interpretação, segundo Minayo (2014).

O projeto foi aprovado pelo Comitê de Ética em Pesquisa da Universidade Estadual do Oeste do Paraná, sob o parecer: 3.801.251 - CAAE: 25286919.0.0000.0107 e atende às normas da Resolução 466/2012, que envolve pesquisa com seres humanos.

\section{Resultados}

Os participantes desta pesquisa foram sete mulheres e quatro homens, com idade entre 25 a 61 anos (média de 42 anos), apresentando despigmentação em várias regiões do corpo, sendo a face a região mais atingida entre os participantes, conforme mostra o Quadro 1. 
Quadro 1: Características do vitiligo, conhecimento sobre a planta, forma de tratamento e resultados. Foz do Iguaçu, PR, Brasil, 2020.

\begin{tabular}{|c|c|c|c|c|c|c|c|}
\hline $\mathrm{P}$ & $\begin{array}{l}\text { Região do } \\
\text { corpo } \\
\text { afetada }\end{array}$ & $\begin{array}{c}\text { Como } \\
\text { Conheceu a } \\
\text { planta }\end{array}$ & $\begin{array}{l}\text { Tempo } \\
\text { de uso }\end{array}$ & $\begin{array}{c}\text { Quantidade planta } \\
\text { utilizada na } \\
\text { infusão }\end{array}$ & $\begin{array}{l}\text { Ingestão } \\
\text { infusão/dia }\end{array}$ & $\begin{array}{l}\text { Tempo de uso } \\
\text { que observou } \\
\text { pigmentação }\end{array}$ & $\begin{array}{c}\text { Observações do } \\
\text { indivíduo quanto a } \\
\text { mancha }\end{array}$ \\
\hline 1 & $\begin{array}{l}\text { Face } \\
\text { Braços e } \\
\text { mãos }\end{array}$ & $\begin{array}{l}\text { Outros } \\
\text { portadores de } \\
\text { vitiligo }\end{array}$ & $\begin{array}{c}10 \\
\text { meses }\end{array}$ & $\begin{array}{l}5 \text { folhas em } 1 \\
\text { L/água }\end{array}$ & $1 \mathrm{~L}$ & 3 meses & $\begin{array}{l}\text { Pigmentação da } \\
\text { área } \\
\text { despigmentada }\end{array}$ \\
\hline 2 & $\begin{array}{c}\text { Face, } \\
\text { abdome e } \\
\text { dorso }\end{array}$ & Revista & 8 meses & $\begin{array}{c}5 \text { folhas em } 1 \\
\text { L/água }\end{array}$ & $1 \mathrm{~L}$ & 4 meses & $\begin{array}{l}\text { Pigmentação da } \\
\text { área } \\
\text { despigmentada }\end{array}$ \\
\hline 3 & Face & $\begin{array}{c}\text { Outros } \\
\text { portadores de } \\
\text { vitiligo } \\
\end{array}$ & 7 meses & $\begin{array}{l}4 \text { folhas em } 11 \\
\text { L/água }\end{array}$ & $800 \mathrm{~mL}$ & 4 meses & $\begin{array}{l}\text { Pigmentação da } \\
\text { área } \\
\text { despigmentada }\end{array}$ \\
\hline 4 & $\begin{array}{l}\text { Face, tórax } \\
\text { e pernas }\end{array}$ & $\begin{array}{l}\text { Outros } \\
\text { portadores de } \\
\text { vitiligo }\end{array}$ & $\begin{array}{c}11 \\
\text { meses }\end{array}$ & $\begin{array}{c}5 \text { folhas em } 1 \\
\text { L/água }\end{array}$ & $1 \mathrm{~L}$ & 3 meses & $\begin{array}{l}\text { Pigmentação da } \\
\text { área } \\
\text { despigmentada }\end{array}$ \\
\hline 5 & Face & Internet & $\begin{array}{c}13 \\
\text { meses }\end{array}$ & $\begin{array}{l}5 \text { folhas em } 1 \\
\text { L/água }\end{array}$ & $500 \mathrm{~mL}$ & 4 meses & $\begin{array}{l}\text { Pigmentação da } \\
\text { área } \\
\text { despigmentada }\end{array}$ \\
\hline 6 & Pernas & $\begin{array}{c}\text { Outros } \\
\text { portadores de } \\
\text { vitiligo }\end{array}$ & 8 meses & $\begin{array}{l}4 \text { folhas em } 1 \\
\text { L/água }\end{array}$ & $1 \mathrm{~L}$ & 4 meses & $\begin{array}{l}\text { Pigmentação da } \\
\text { área } \\
\text { despigmentada }\end{array}$ \\
\hline 7 & Face e tórax & Internet & $\begin{array}{c}12 \\
\text { meses }\end{array}$ & $\begin{array}{c}4 \text { folhas em } 1 \\
\text { L/água }\end{array}$ & $500 \mathrm{~mL}$ & 3 meses & $\begin{array}{c}\text { Pigmentação da } \\
\text { área } \\
\text { despigmentada }\end{array}$ \\
\hline 8 & $\begin{array}{l}\text { Dorso das } \\
\text { mãos }\end{array}$ & Internet & 3 meses & $\begin{array}{c}5 \text { folhas em } 1 \\
\text { L/água }\end{array}$ & $200 \mathrm{~mL}$ & 3 meses & $\begin{array}{l}\text { Pigmentação } \\
\text { parcial da área } \\
\text { despigmentada }\end{array}$ \\
\hline 9 & $\begin{array}{l}\text { Pernas e } \\
\text { braços }\end{array}$ & Internet & $\begin{array}{c}12 \\
\text { meses }\end{array}$ & $\begin{array}{l}5 \text { folhas em } 1 \\
\text { L/água }\end{array}$ & $500 \mathrm{~mL}$ & 4 meses & $\begin{array}{l}\text { Pigmentação da } \\
\text { área } \\
\text { despigmentada }\end{array}$ \\
\hline $\begin{array}{l}1 \\
0\end{array}$ & Face e tórax & $\begin{array}{c}\text { Outros } \\
\text { portadores de } \\
\text { vitiligo }\end{array}$ & 7 meses & $\begin{array}{l}2 \text { folhas em } \\
500 \mathrm{~mL} / a ́ g u a\end{array}$ & $500 \mathrm{~mL}$ & 4 meses & $\begin{array}{c}\text { Pigmentação da } \\
\text { área } \\
\text { despigmentada }\end{array}$ \\
\hline $\begin{array}{l}1 \\
1\end{array}$ & $\begin{array}{l}\text { Tórax e } \\
\text { dorso }\end{array}$ & $\begin{array}{c}\text { Outros } \\
\text { portadores de } \\
\text { vitiligo } \\
\end{array}$ & $\begin{array}{c}10 \\
\text { meses }\end{array}$ & $\begin{array}{c}5 \text { folhas em } 1 \\
\text { L/água }\end{array}$ & $1 \mathrm{~L}$ & 6 meses & $\begin{array}{l}\text { Pigmentação da } \\
\text { área } \\
\text { despigmentada }\end{array}$ \\
\hline
\end{tabular}

Fonte: Dados da pesquisa.

Ainda pelo Quadro 1, é possível observar que os participantes descreveram ter conhecido a planta por meio de outros portadores de vitiligo, internet e revistas.

O grupo estudado fazia uso da infusão das folhas da SO no período entre 3 e 13 meses (média de 9 meses), preparando o chá com uma média de 5 folhas em um litro de água, consumindo em média $700 \mathrm{~mL}$ no decorrer do dia. Quanto a característica das manchas pelo corpo, dez indivíduos observaram pigmentação das áreas despigmentadas e um observou pigmentação parcial das manchas, talvez pelo pouco tempo e quantidade ingerida da infusão. A pigmentação das áreas foi observada no período entre 3 e 6 meses após o início do uso da infusão (média de 4 meses).

Com respeito aos sentimentos, as narrativas dos participantes traduzem os principais aspectos relacionados a sua imagem corporal e os sentimentos por conviver com o vitiligo.

Faz muitos anos que tenho, me sentia feio (P1).

Fiquei triste desde quando iniciou as manchas (P3).

Tinha vergonha de usar saia ou shorts, ficava triste (P6). 
Uma mulher com o rosto cheio de manchas, ah, isso é triste (P7).

Ter essas manchas me aborrece tanto (P8).

Essas manchas assim pelo corpo né, me sentia diferente (P11).

A imagem corporal abalada pode traduzir efeitos prejudiciais a curto e longo prazo, em especial para a mulher, podendo levar a sentimento de tristeza, aborrecimento e diferença, com impacto direto na vida cotidiana, considerando a valorização da aparência e da expressão do corpo para as relações interpessoais.

Com a evolução de tratamentos naturais do vitiligo a base de SO, as participantes foram percebendo que as manchas decorrentes a doença foram desaparecendo, neste momento, suas percepções voltaram-se para sentimentos mais positivos.

Muito contente, até que enfim as manchas estão sumindo (P2).

Que maravilha ver as manchas sumindo, fiquei muito feliz (P3).

Tentei tantas coisas, até que enfim esse deu certo, estou feliz (P5).

Estou satisfeita, está indo, né? (P6)

Nem me fale [fica pensativa], tentei remédios [se emociona], até reza tentei. E deu certo agora, estou feliz (P7).

Não acreditava muito, mas está dando certo, estou satisfeita (P8).

Com o passar dos anos e com as inúmeras tentativas de tratamento nada exitosas, os participantes foram desacreditando que sua aparência física pudesse mudar e que sua vida pudesse voltar ao normal, recorrendo a uma diversidade de remédios e a espiritualidade. Contudo, a partir do momento que observaram melhoras com a utilização da planta, sentimentos de felicidade e gratidão foram reascendendo a esperança de uma vida melhor.

\section{Discussão}

A planta medicinal SO, conhecida popularmente no Brasil como Serralha, tem demonstrado aplicações terapêuticas importantes, como em distúrbios gastrointestinais (Alothman et al., 2018), anti-inflamatória, antidiabético, antioxidante, antipirético (Puri et al., 2018), diurético, cura para doenças hepáticas, anemia e cicatrizante (Nonato et al., 2018).

Além destas propriedades, contemporaneamente, alguns estudos indicam a possibilidade da SO também auxiliar no tratamento de Vitiligo por observar a pigmentação da pele em áreas despigmentadas de indivíduos portadores da doença que consumiam a infusão desta planta medicinal (Nonato et al., 2018; Resende et al., 2015).

No presente estudo, foi relatado o uso da infusão da SO por 11 indivíduos portadores de vitiligo, que faziam uso diariamente de aproximadamente $700 \mathrm{~mL}$ da infusão da SO, após preparar em torno de $1 \mathrm{~L}$ do chá com 5 folhas da planta em média e consumiam essa infusão por 9 meses (média).

Os participantes verbalizaram a pigmentação da área afetada pelo vitiligo, em um período médio de 4 meses de uso da infusão. Estes dados corroboram com a pesquisa de Resende e seus colaboradores (2015), que acompanharam por um ano 12 portadores de vitiligo que consumiram a infusão da SO e indicaram a SO como uma planta medicinal com propriedades para auxiliar no tratamento do vitiligo.

Plantas medicinais que auxiliam no tratamento do vitiligo podem estar associadas a produção de melanina, visto que, a melanina é uma proteína responsável pela importante função de proteção da pele humana contra a radiação ultravioleta e também por dar pigmentação aos cabelos, olhos e pele, sendo produzida pelos melanócitos por meio da melanogênese. A principal causa do vitiligo é a destruição de melanócitos, logo, acredita-se que a pigmentação das áreas afetadas pelo vitiligo seja ocasionada por um ou mais agente que estimule a produção de melanogênese e, consequentemente, a produção de 
melanina (Niu \& Aisa, 2017).

Em uma revisão sobre os aspectos farmacológicos e fitoquímicos da SO, é apresentado que esta planta possui compostos químicos como: Sesquiterpenos lactonas, flavonóides, fenóis totais, cetonas, saponinas e proantocianidinas (Puri et al., 2018). Pesquisas indicam que a maioria destes compostos possuem a capacidade de ativar a produção de melanogênese; Sesquiterpenos (Mamoon et al., 2019), flavonóides e cetonas (Niu et al., 2016), podendo então ser estes componentes químicos da SO os responsáveis pela repigmentação das manchas brancas nos portadores de vitiligo.

$\mathrm{O}$ vitiligo pode se manifestar com sintomas leves, ocasionando pequenas manchas brancas pelo corpo, ou causar despigmentação grave, deixando o indivíduo com poucas manchas da cor de sua pele espalhadas pelo corpo (Niu \& Aisa, 2017). Embora o objetivo do tratamento é a repigmentação das áreas afetadas, o resultado pode ser considerado positivo quando ocorre a despigmentação das poucas áreas que não foram afetadas pelo vitiligo, quando ele se manifesta de forma grave. A despigmentação da pele se dá pela proantocianidinas, composto químico presente na SO e que possui efeito inibitório na melanogênese (Fujimaki et al., 2018). A despigmentação total da pele é mais tolerável do que a pele manchada que sujeita o indivíduo a estigmas e preconceitos (Resende et al., 2015).

Com respeito aos sentimentos relacionados a sua aparência devido ao vitiligo, os participantes relataram sentimentos negativos, sendo a tristeza a mais comum, seguida de sentir-se aparentemente feio. Pesquisadores apontam que o portador de vitiligo sofre impactos psicológicos devastadores (Lai et al., 2017), sugerindo uma ligação entre vitiligo e morbidade psiquiátrica.

Os sintomas depressivos recorrentes nos discursos dos portadores de vitiligo desta pesquisa, proferidos principalmente pela tristeza, indicam claramente o extenso sofrimento psicológico que eles enfrentam, somado aos sentimentos de estresse, ansiedade e baixo autoestima, considerando que esses indivíduos não se sentem autoconfiantes devido a sua aparência (Simons et al., 2020). Importante destacar que todos esses sintomas depressivos demonstram uma conexão fisiopatológica (Kota et al., 2019; Vallerand et al., 2019) podendo resultar no agravamento da doença, ou ainda, a outros problemas de saúde física.

$\mathrm{O}$ vitiligo pode ser considerado uma doença grave ao se analisar seu impacto negativo em um relacionamento afetivo, procura de emprego, rejeição social, na dificuldade de escolher uma roupa como apontado na narrativa de uma participante e também na dificuldade de frequentar eventos sociais (Kota et al., 2019). O impacto na saúde mental destes indivíduos ocorre em decorrência da visibilidade dos distúrbios na pele, que transcende a percepção de beleza, raça, cor, idade e condições econômicas (Grimes \& Miller, 2018).

Após a utilização da infusão da SO, os integrantes deste estudo indicaram que se sentiam felizes e satisfeitos, mediante a dissociação de sua imagem a uma doença. Resultados similares foram observados em portadores de vitiligo que receberam camuflagem cosmética, indicando que a ausência das manchas traz uma melhor qualidade de vida (Salsberg et al., 2016).

Nenhum indivíduo relatou efeitos colaterais quanto ao uso da infusão da SO. Estudos em avaliação da toxicidade desta planta não apontam alterações hematológicas ou anatomopatológica. Esses dados conferem segurança para o uso de formulações a base da SO (Nonato et al., 2018; Alothman et al., 2018).

Há de ressaltar que o número de participantes desta pesquisa oferece limitações a este estudo, sendo que, mais participantes poderiam oferecer maiores informações quanto a ação da SO no tratamento do vitiligo, assim como, sobre a segurança de seu uso.

\section{Conclusão}

A presente investigação mostra que a infusão de SO ocasionou a pigmentação das manchas brancas de portadores de vitiligo, assim como, os resultados da terapia fitoterápica afastaram os sentimentos negativos, proporcionando uma melhor 
qualidade de vida. O desfecho deste estudo também indica que a SO pode servir no desenvolvimento de novos medicamentos e apoio para novas pesquisas. Assim, sugere-se que novas pesquisa sobre os efeitos do SO sejam realizadas não somente com a infusão desta planta medicinal, mas também com seu extrato, creme ou pomada.

\section{Referências}

Alothman, E. A., Awaad, A. S, Safhi, A. A., Almoqren, S. S., El-Meligy, R. M., Zain, Y. M, Alasmary, A. S. \& Alqasoumi, S. I. (2018). Evaluation of antiulcer and ulcerative colitis of Sonchus oleraceus L. Saudi Pharmaceutical Journal, 26(1), 956-959.

Dalla, A., Parsad, D., Vinay, K., Thakur, V. \& Kumaran, M. S. (2020). A prospective study to assess the efficacy of various surgical modalities in treatment of stable vitiligo patches over resistant sites. International journal of dermatology, 59(7), 837-842.

Doulah, A., Mahmoodi, G. \& Borujeni, M. P. (2020). Evaluation of the pre-treatment effect of centella asiatica medicinal plants on long-term potentiation (LTP) in rat model of Alzheimer's disease, Neuroscience letters, 11(729), 135026.

Fujimaki, T., Mori, S., Horikawa, M. \& Fukui, Y. (2018). Isolation of proanthocyanidins from red wine, and their inhibitory effects on melanin synthesis in vitro. Food chemistry, 15(248), 61-69.

Grimes, P. E. \& Miller, M. M. (2018). Vitiligo: patient stories, self-esteem, and the psychological burdenof disease. International Journal of Women's Dermatology, 4(1), 32-37.

Kapoor, R., Dhatwalia, S. K., Kumar, R., Rani, S. \& Parsad, D. (2020). Emerging role of dermal compartment in skin pigmentation: comprehensive review. Journal of the European Academy of Dermatology and Venereology, 34(12), 2757-2765.

Kota, R. S., Vora, R.V., Varma, J. R., Kota, S. K., Patel, T. M. \& Ganjiwale, J. (2019). Study on assessment of quality of life and depression in patients of vitiligo. Indian Dermatology Online Journal, 10(2), 153-157.

Lai, Y. C., Yew, Y. W., Kennedy, C. \& Schwartz, R. A, (2017). Vitiligo and depression: a systematic review and meta-analysis of observational studies. The British journal of dermatology, 177(3), 708-718.

Mamoon, U., Alamzeb, R. M., Ali, S., Ullah, S., Shah, A. Z, Naz, I. \& Khan, M. R. (2019). The chemistry and pharmacology of alkaloids and allied nitrogen compounds from artemisia species: a review. Phytotherapy research, 33(10), 2661-2684.

Mattos, G., Camargo, A., Sousa, C. A. \& Zeni, A. L. B. (2018). Medicinal plants and herbal medicines in primary health care: the perception of the professionals. Ciência \& Saúde Coletiva, 23(11), 3735-3744.

Minayo, M. C. S. (2014). O desafio do conhecimento: pesquisa qualitativa em saúde. (14a ed.), Hucitec-Abrasco.

Ministério da Saúde (BR). (2006a). Secretaria de Assistência à Saúde. Departamento de Atenção Básica. Política Nacional de Práticas Integrativas e Complementares no SUS: PNPIC-SUS. http://bvsms.saude.gov.br/bvs/publicacoes/politica_nacional_praticas_integrativas_complementares_2ed.pdf

Ministério da Saúde (BR). (2006b). Secretaria de Ciência, Tecnologia e Insumos Estratégicos. Departamento de Assistência Farmacêutica. Política Nacional de Plantas Medicinal e Fitoterápicos. http://bvsms.saude.gov.br/bvs/publicacoes/politica_nacional_fitoterapicos.pdf.

Niu, C. \& Aisa, H. A. (2017). Upregulation of melanogenesis and tyrosinase activity: potential agents for Vitiligo. Molecules, $22(8), 1303$.

Niu, C., Yin, L., Nie, L. F., Dou, J., Zhao, J. Y, Li, G. \& Aisa, H. A. (2016). Synthesis and bioactivity of novel isoxazole chalcone derivatives on tyrosinase and melanin synthesis in murine B16 cells for the treatment of vitiligo. Bioorganic \& medicinal chemistry, 24(21), 5440-5448.

Nonato, I. A., Viloria, M. I. V., Carvalho, G. D., Valente, F. L., Salcedo, J. H. P., Rosa, M. B. \& Carvalho, C. A. (2018). Healing effects of formulations with extract of Sonchus oleraceus. Acta Scientiae Veterinariae, 46(1), 1605.

Oliveira, A.F.P., Costa, I.C.P., Andrade, C.G., Santos, K.F.O. \& Anízio, B. K. F. (2017). Fitoterapia na atenção básica: estudo com profissionais enfermeiros. Revista online de Pesquisa Cuidado é Fundamental, 9(2), 480-487.

Pereira, A. S., Shitzuka, D. M., Parreira, F. J. \&Shitzuka, R. (2018). Metodologia da pesquisa científica. UFSM.

Puri, A. V., Khandagale, P. D. \& Ansari, Y. N. (2018). A review on ethnomedicinal, pharmacological and phytochemical aspects of Sonchus oleraceus linn. (Asteraceae). International Journal of Pharmacy and Biological Sciences, 8(3), 1-9.

Resende, J. H. C., Aquino, G. S. T., Nascimento, F. R. F., Aguiar, M. M. \& Fiorelli, R. K. A. (2015). Oral use of an infusion of leaves of Solanum paniculatum L., Jacaranda brasiliensis and Sonchus oleraceus for treatment of vitiligo. Dermatological Sciences and Applications, 5, 317-331.

Rodríguez, M. A., Geertsma, I. P., Françozo, M. \& Van, A. T. (2020). Marcgrave and piso's plants for sale: the presence of plant species and names from the história naturalis brasiliae (1648) in contemporary Brazilian markets. Journal of Ethnopharmacol, 7(1), 112911.

Salsberg, J. M., Weinstein, M., Shear, N., Lee, M. \& Pope, E. (2016). Impact of cosmetic camouflage on the quality of life of children with skin disease and their families. Journal of cutaneous medicine and surgery, 20(3), 211-215.

Shah, B., Godse, K., Mahajan, S., Grandhi, S., Shendkar, S., Sharma, A., Teli, C., Pathak, R. \& Parsad, D. (2019). Efficacy and safety of basic fibroblast growth factor (bFGF) related decapeptide solution plus Tacrolimus $0.1 \%$ ointment versus Tacrolimus $0.1 \%$ ointment in the treatment of stable vitiligo. Dermatologic therapy, 32(6), e13109. 
Research, Society and Development, v. 10, n. 4, e1410413824, 2021

(CC BY 4.0) | ISSN 2525-3409 | DOI: http://dx.doi.org/10.33448/rsd-v10i4.13824

Simons, R. E., Zevy, D. L. \& Jafferany, M. (2020). Psychodermatology of vitiligo: psychological impact and consequences. Dermatologic Therapy, 33(3), e13418

Vallerand, I. A., Lewinson, R. T., Parsons, L. R., Hardin, J., Haber, R. M., Lowerison, M. W., Barnabe, C. \& Patten S. B. (2019). Vitiligo and major depressive disorder: a bidirectional population-based cohort study. Journal of the American Academy of Dermatology, 80(5), 1371-1379.

Zeni, A. L. B., Parisotto, A. V., Mattos, G. \& Helena, E. T. S. (2017). Use of medicinal plants as home remedies in primary health care in Blumenau - state of Santa Catarina, Brazil. Ciência \& Saúde Coletiva, 22(8), 2703-2712. 